\title{
A note on a paper of Williamson
}

\section{J.L. Lavoie and R. Tremblay}

An elementary proof is given of a result obtained by concerning the sum of an infinite series involving a ${ }_{4} F_{3}$.

\section{Introduction and main result}

Recently Williamson [2] obtained the sum of an infinite series involving the ${ }_{4} F_{3}$ function in terms of gamma functions (see (3), below)).

This result was derived from a formula for an infinite series involving the product of two Bessel functions of the first kind, by utilising the integral representation for the product together with Poisson's summation formula and the Weber-Schafheitlin discontinuous integral.

The restrictions on the parameters $a$ and $c$ appearing in this summation formula are stated to be $2 c+\frac{1}{2}>2 a>1$. These inequalities are certainly satisfied for $a=c=1$ and $a=c=3 / 4$, and yet it is readily verified that the ${ }_{4} F_{3}$ series diverges when the summation index is equal to 1 . A gamma function is also missing from the right-hand side of Williamson's result. This fact becomes clear from the more general result

$$
\text { (1) } \begin{aligned}
\sum_{m=0}^{\infty}\left(\frac{n+1}{m+\alpha}\right)^{a+1}{ }_{4} F_{3}\left(\begin{array}{r}
a / 2+1 / 2, a / 2+1, b / 2+1 / 2, b / 2+1 \\
3 / 2, b+1 / 2, b+1
\end{array}\right. & \left.\mid\left(\frac{n+1}{m+a}\right)^{2}\right) \\
& =\frac{1}{a} \sum_{m=0}^{n}\left(\frac{n+1}{m+\alpha}\right)_{2}^{a} F_{1}\left(\begin{array}{l}
a, b \\
2 b
\end{array} \mid \frac{n+1}{m+\alpha}\right),
\end{aligned}
$$

where $n=0,1,2, \ldots, \alpha$ is a positive number greater than $n+1$,

Received 3 January 1975. This work was supported by a grant from the National Research Counc il of Canada. 
and $a>0$. If $\alpha=n+I$ the restriction $b>a$ must be added. The proof of this result is elementary.

\section{Proof}

The right-hand side of (1) can be written

$$
\frac{1}{a}\left\{\sum_{m=0}^{\infty}\left(\frac{n+1}{m+\alpha}\right)^{a} 2^{F_{1}}\left(\begin{array}{c|c}
a, b & \frac{n+1}{m+\alpha}
\end{array}\right)-\sum_{m=n+1}^{\infty}\left(\frac{n+1}{m+\alpha}\right)^{\alpha} 2_{1}{ }_{1}\left(\begin{array}{c|c}
a, b & \frac{n+1}{m+\alpha}
\end{array}\right)\right\} .
$$

Shifting the summation index so that the sum on the right is from $m=0$ to $m=\infty$ and applying Euler's transformation $[1, \mathrm{p} .60]$ to the $2_{1}$, we obtain

$$
\frac{1}{a} \sum_{m=0}^{\infty}\left(\frac{n+1}{m+\alpha}\right)^{\alpha}\left\{2^{F_{1}}\left(\begin{array}{c|c}
a, b & \frac{n+1}{m+\alpha}
\end{array}\right)-2^{F_{1}}\left(\begin{array}{c|c}
a, b & -\frac{n+1}{m+\alpha}
\end{array}\right)\right\}
$$

this is readily transformed into the left-hand side of (1) by taking out the common factor in the difference between the two ${ }_{2} F_{1}$ 's.

\section{Some special cases}

For $n=0$ the right side of (I) reduces to

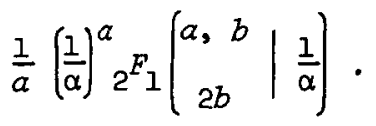

Gauss's summation theorem [1, p. 49] can be used when $\alpha=1$ to yield

$$
\sum_{m=0}^{\infty}\left(\frac{1}{m+1}\right)^{a+1}{ }_{4}{ }_{3}\left(\begin{array}{c|c}
a / 2+1 / 2, a / 2+1, b / 2+1 / 2, b / 2+1 \\
3 / 2, b+1 / 2, b+1
\end{array} \mid\left(\frac{1}{m+1}\right)^{2}\right)=\frac{1}{a} \frac{\Gamma(2 b) \Gamma(b-a)}{\Gamma(b) \Gamma(2 b-a)},
$$

with $b>a>0$.

This is essentially Williamson's result. To see this, replace $a$ and $b$ by $2 a-1$ and $2 b-1$, shift the summation index, and use the duplication formula for the gamma function to obtain

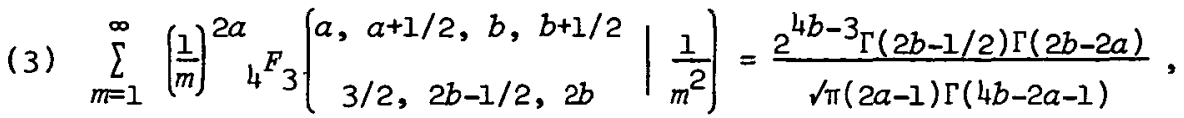


Note that the factor $\Gamma(2 b-2 a)$ has been omitted in Williamson's paper.

There are a few other cases in which the ${ }_{2} F_{1}$ in (2) can be evaluated in terms of gamma functions. For example, if $\alpha=2$ and $b=\frac{1}{3}(a+1)$, the formula [1, p. 69, Exercise 2],

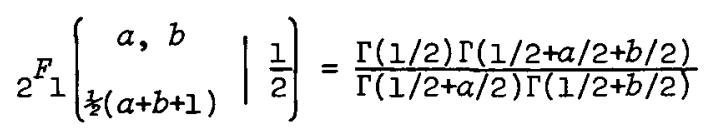

can be employed; this yields

$$
\begin{aligned}
\sum_{m=0}^{\infty}\left(\frac{1}{m+2}\right)^{a+1}{ }_{4} F_{3}\left(\begin{array}{c|c}
a / 2+1 / 2, a / 2+1, a / 6+2 / 3, a / 6+7 / 6 \\
3 / 2, a / 3+5 / 6, a / 3+4 / 3
\end{array}\right. & \left.\left(\frac{1}{m+2}\right)^{2}\right) \\
& =\frac{\Gamma(a / 2+1) \Gamma(2 a / 3+2 / 3)}{a \Gamma(a+1) \Gamma(a / 2+2 / 3)} .
\end{aligned}
$$

Finally let $a=b=1$ in (1). Since $2^{F_{1}}(1,1 ; 2 ; z)=z^{-1} \ln (1-z)$, we have the interesting relation

$$
\prod_{m=0}^{\infty}\left(1-\left(\frac{n+1}{m+\alpha}\right)^{2}\right)=\prod_{m=0}^{n}\left(1-\frac{n+1}{m+\alpha}\right), \quad \alpha>n+1 .
$$

This can be proved by an elementary calculation.

\section{References}

[1] Earl D. Rainville, Special functions (Macmillan, New York, 1960).

[2] A.G. Williamson, "A note on the summation of an infinite series involving a hypergeometric function", Bull. Austral. Math. Soc. $10(1974), 305-309$.

Département de Mathématique,

Université Laval,

Cité Universitaire,

Québec,

Canada;
Département de Physique, Université Laval, Cité Universitaire, Québec, Canada. 